\title{
Alcian Blue and Periodic Acid Schiff Staining Method
}

National Cancer Institute

\section{Source}

National Cancer Institute. Alcian Blue and Periodic Acid Schiff Staining Method. NCI

Thesaurus. Code 192304.

A microscopy staining method that utilizes periodic acid, an oxoacid of iodine, Schiff reagent, the reaction product of fuchsine and sodium bisulfite, and Alcian blue, a water soluble copper phthalocyanine dye. This staining method is used to differentially stain glycoproteins. 